\title{
Laser-capillary interaction for the EXIN project
}

\author{
F.G. Bisesto ${ }^{\mathrm{a}, \mathrm{b}, *}$, M.P. Anania ${ }^{\mathrm{a}}$, A.L. Bacci ${ }^{\mathrm{c}}$, M. Bellaveglia $^{\mathrm{a}}$, E. Chiadroni $^{\mathrm{a}}$, A. Cianchi ${ }^{\mathrm{a}, \mathrm{d}}$, \\ A. Curcio ${ }^{\mathrm{a}, \mathrm{b}}$, D. Di Giovenale ${ }^{\mathrm{a}}$, G. Di Pirro ${ }^{a}$, M. Ferrario $^{\mathrm{a}}$, M. Galletti ${ }^{\mathrm{a}}$, A. Gallo ${ }^{\mathrm{a}}$, A. Ghigo ${ }^{\text {a }}$, \\ A. Marocchino ${ }^{\text {b,e }}$, A. Mostacci ${ }^{\text {b,e }}$, M. Petrarca ${ }^{\text {bee }}$, R. Pompili ${ }^{a}$, A.R. Rossi ${ }^{c}$, L. Serafini $^{c}$, \\ C. Vaccarezza ${ }^{\text {a }}$ \\ a INFN-LNF, Via Enrico Fermi 40, 00044 Frascati, Italy \\ b Sapienza - University of Rome, P.le Aldo Moro, 2, 00185 Rome, Italy \\ c INFN - Milano, Via Celoria 16, 20133 Milan, Italy \\ d Universit degli studi di Roma Tor Vergata, Via di Tor Vergata, Rome, Italy \\ e INFN - Roma1, P.le Aldo Moro, 2, 00185 Rome, Italy
}

\section{A R T I C L E I N F O}

Available online 22 January 2016

Keywords:

High power lasers

Ultrashort laser pulse

Capillary

Plasma wake field acceleration

Optics

\begin{abstract}
A B S T R A C T
The EXIN project is under development within the SPARC_LAB facility of the National Laboratory of Frascati (LNF-INFN). This project aims to accelerate pre-existing electron bunches with high brightness by exploiting the wakefield plasma acceleration technique, while preserving the initial brightness. The wakefield is excited inside a dielectric capillary by high intensity laser pulses produced by the FLAME laser interacting with a gas. In this work, we present numerical simulations in order to optimize energy coupling between our laser with super-Gaussian transverse profile and a dielectric capillary. Moreover, an overview of the experimental layout will be given.
\end{abstract}

(c) 2016 Elsevier B.V. All rights reserved.

\section{Introduction}

During the last 15 years, there has been an enormous interest towards the novel particles acceleration scheme based on laserplasma interaction and the main reason resides on the possibility to generate high longitudinal electric field gradients by the interaction of an intense laser pulse with a plasma. These gradients can reach $\sim 100 \mathrm{GV} / \mathrm{m}$ in the linear regime and several hundreds $\mathrm{GV} / \mathrm{m}$ in the non-linear regime $[1,2]$, therefore exceeding more than a factor of 1000 the fields provided by conventional RF structures.

In order to obtain higher energies, it is necessary to extend the accelerating length as much as possible. Therefore, the main limitations due to the short Rayleigh length of the laser and due to the dephasing mechanism have been investigated. A possible solution to overcome them is to use gas-filled dielectric capillaries [3-5] and/or by tailoring the density of the plasma [6] while to overcome the depletion effect [7] a staging scheme has been proposed [8]. Despite the high energy requirement, most of the applications that can benefit from this novel acceleration scheme demands high brightness particle bunches. Therefore, the EXIN project is dedicated to exploit

\footnotetext{
* Corresponding author: Tel. +39069403 8077.

E-mail address: fabrizio.giuseppe.bisesto@Inf.infn.it (F.G. Bisesto).
}

the wakefield acceleration with particular care to the quality of the accelerated bunches. In this scheme, the wakefield will be created by the FLAME laser pulse [9] propagating in gas filled capillary and it will be used to accelerate the electron bunches produced by the high brightness SPARC photoinjector. The main advantages of this scheme are twofold: first of all the electron bunch characteristics (emittance, energy spread, charge, etc.) are already well defined prior the wakefield acceleration stage and then, since the bunch generation is decoupled by the acceleration process, it might be possible to have more control on the injection mechanism i.e. the injection of the electron bunches into the accelerating field. Mastering this process is of crucial importance to preserve the initial quality of the bunches during the acceleration.

\section{Electromagnetic field modes in a dielectric capillary}

A dielectric capillary is an hollow fiber characterized by an empty internal core and an external glass wall, commonly made in borosilicate. This kind of waveguide is different from an optical fiber, where the core has a greater refractive index than the cladding. For this reason it exploits total internal reflection to guide a laser beam. Since the capillary does not have this 
characteristic, a laser beam will experience more losses during the propagation.

The modes of the hollow waveguide are calculated using the propagation equation for a homogeneous dielectric medium

$\nabla^{2} \mathbf{E}-\frac{\epsilon}{c^{2}} \frac{\partial^{2} \mathbf{E}}{\partial t^{2}}=0$

where $\mathbf{E}$ is the electric field, $\epsilon$ is the relative dielectric constant and $c$ is the speed of light in vacuum. Eq. (1) can be solved in cylindrical coordinates for the $z$-component of electric field, since the other two components are related to it by Maxwell's equations [10]. The solution of Eq. (1) takes the form $E_{z}(\mathbf{r}, t)=E_{z}(r) e^{i\left(\nu \theta+\omega t-k_{z} z\right)}$, where $\theta$ is the angle in the transverse plane, $k_{z}$ is the $z$-component of the wave vector and $\nu$ is an integer.

In particular, TE or TM modes $(\nu=0)$ cannot be properly excited by an incident Gaussian-like beam with linear polarization that, on the

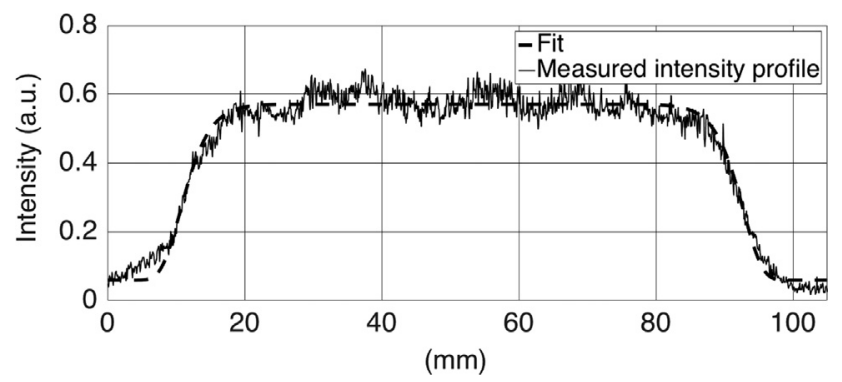

Fig. 1. FLAME measured intensity profile. From a super-Gaussian fit, it figured out $n=8$ and $F W H M=8 \mathrm{~cm}$ other hand, can efficiently be coupled to hybrid modes with $\nu=1$, $E H_{1 m} \propto J_{0}\left(u_{m} \frac{r}{a}\right)$, where $u_{m}$ is the first zero of Bessel function $J_{m}$ [11].

\section{Coupling efficiency between super-Gaussian transverse laser profile and dielectric capillary}

In order to optimize the laser injection into the capillary, the key point is to maximize the amount of energy coupled to the first $E H_{11}$ mode [13]. For an incident Gaussian beam, it has been shown [14] that the fundamental mode $E H_{11}$ can be coupled with the $98 \%$ of its energy if the ratio $\frac{w_{0}}{a}$ between beam waist at focus $w_{0}$ and capillary radius $a$ is equal to 0.645 .

Nevertheless, the FLAME high power lasers (100 TW) is characterized by a super-Gaussian, rather than Gaussian, transverse profile. To find the best energy coupling condition for a superGaussian laser profile, numerical simulations have been performed. The characterization of the FLAME laser intensity (Fig. 1) reveals a super-Gaussian spatial profile of order $n=8$ and a diameter of $F W H M=8 \mathrm{~cm}$ (Fig. 2a). These parameters have been used to calculate the intensity profile at the focal plane by computing the expression

$E_{\text {focus }}(r) \propto \mathcal{F}\left(\rho(r) \cdot e^{\left(-r / w_{i}\right)^{2 n}}\right)$, where $\mathcal{F}$ identifies the Fourier transform operator, $\rho(r)$ is the lens pupil function describing the lens aperture and $w_{i}$ the initial beam radius on the lens calculated at $1 / e^{2}$. The lens aperture has been chosen with a radius equals to $76.2 \mathrm{~mm}$

The beam intensity at focal plane (Fig. 2b) presents two secondary lobes, whose peak intensity is about $1.6 \%$ of the principal one. Since they will hit the cladding wall, with intensities of the order of $10^{16} \mathrm{~W} / \mathrm{cm}^{2}$, these peaks can significantly damage the capillary. To overcome this problem, the use of a tapered capillary
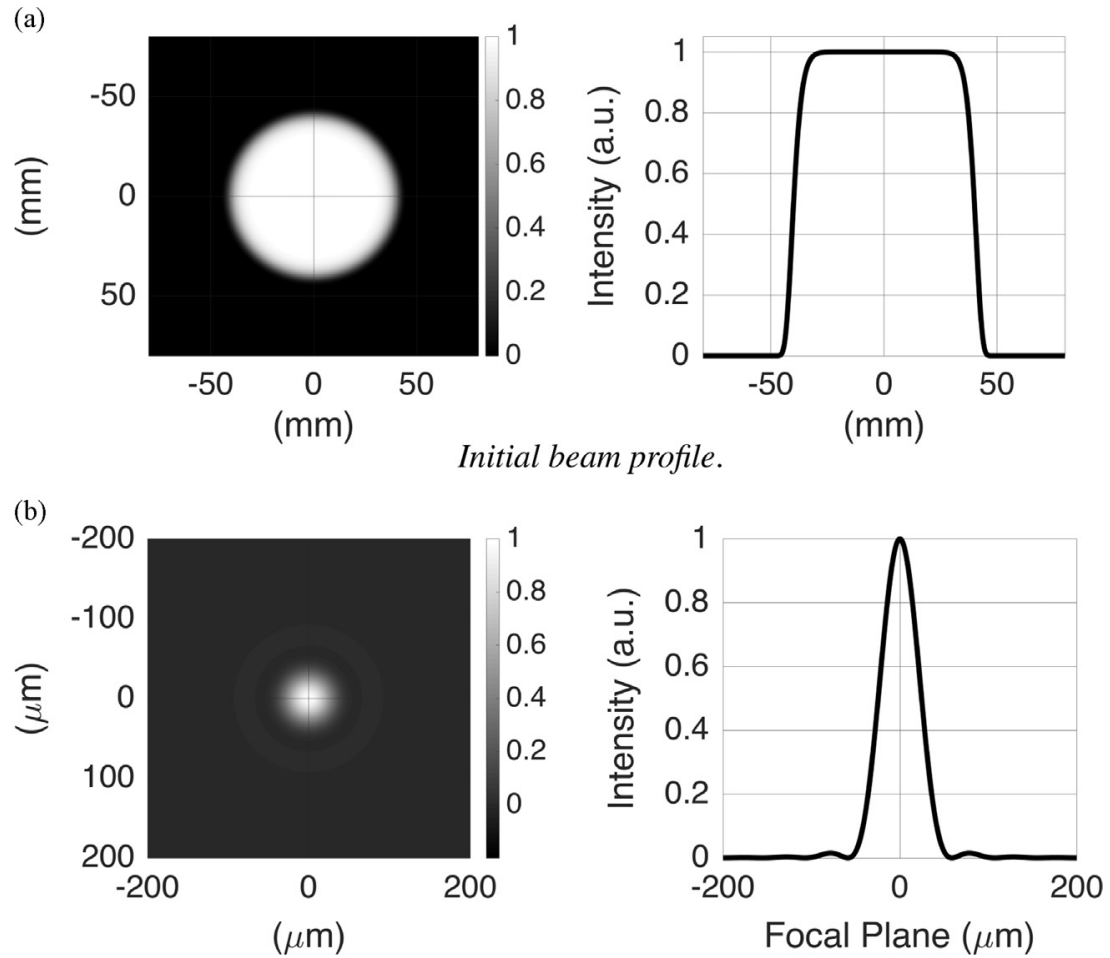

Beam intensity at focal plane.

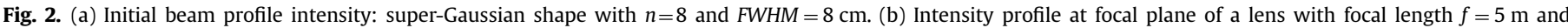
aperture radius $R=76.2 \mathrm{~mm}$. 


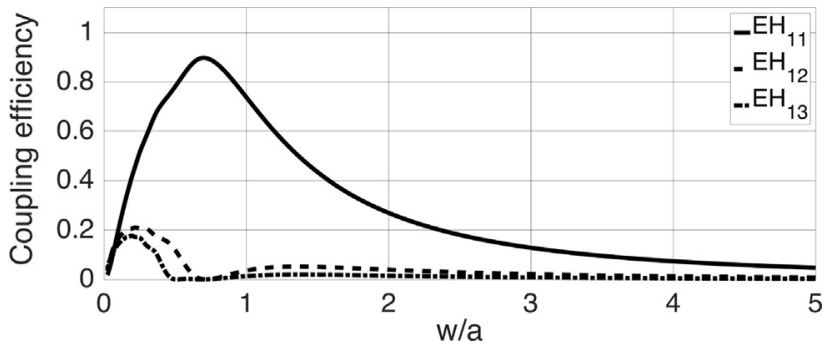

Fig. 3. Energy coupling efficiency between the focused super-Gaussian laser beam and e.m. modes in a dielectric capillary. The best coupling value is equals to $89.80 \%$ for $\frac{w}{a}=0.70$, where $w$ is the radius at $\frac{1}{e^{2}}$ of first lobe of intensity profile. The simulation has been performed for the first three modes $E H_{11}$ (solid line), $E H_{12}$ (dashed line), and $\mathrm{EH}_{13}$ (dash-dot line).

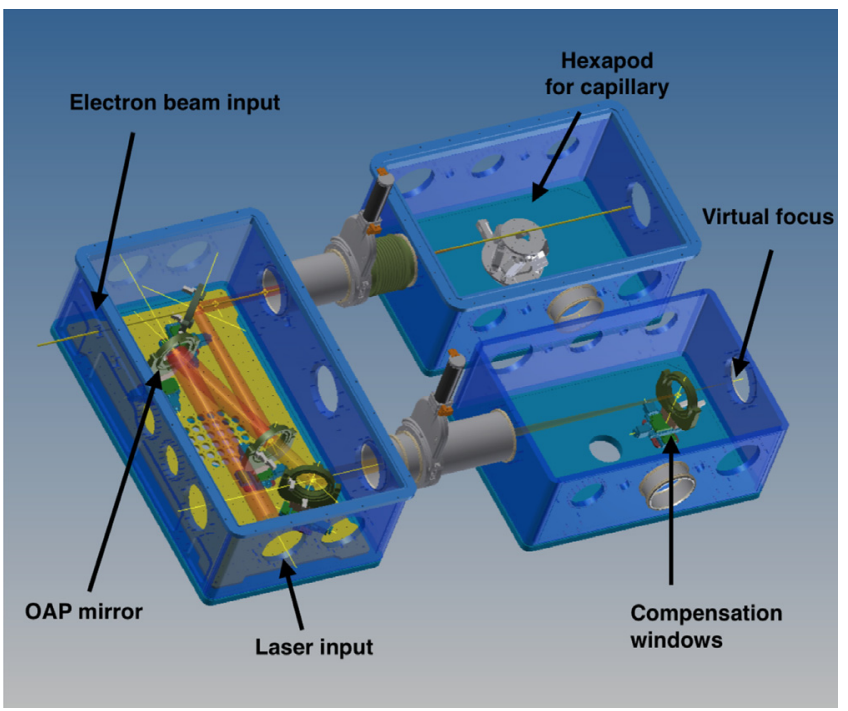

Fig. 4. Layout of EXIN experimental chamber: laser beam will be focused by OAP mirror, while two mirrors are required for the alignment; dielectric capillary will be placed on a PI (Physik Instrumente) hexapod in order to properly align it; a diagnostic chamber will be installed to measure on-line the virtual focus, the laser spectral profile, the plasma spectral emission and other parameters that can characterize the acceleration process.

[12] or the possibility to introduce a ceramic diaphragm, to spatially filter out the secondary lobes, is under study.

The energy coupling efficiency between the fundamental mode $E H_{11}$ and the laser can be computed as

$$
\begin{aligned}
\eta_{m} & =\frac{\int\left|E H_{11} \cdot E_{\text {laser }}\right|^{2} d r}{\int\left|E H_{11}\right|^{2} d r \int\left|E_{\text {laser }}\right|^{2} d r} \\
& =\frac{\int\left|J_{0}\left(u_{m} \frac{r}{a}\right) \cdot \mathcal{F}\left(\rho(r) \cdot e^{\left(-r / w_{0}\right)^{2 n}}\right)\right|^{2} d r}{\int\left|J_{0}\left(u_{m} \frac{r}{a}\right)\right|^{2} d r \int\left|\mathcal{F}\left(\rho(r) \cdot e^{\left(-r / w_{0}\right)^{2 n}}\right)\right|^{2} d r}
\end{aligned}
$$

where $u_{m}$ is the first zero of $m$ th order Bessel function. Simulations have been performed for the first three hybrid modes. As shown in Fig. 3 , the best coupling condition equals to $89.80 \%$ is fulfilled for $\frac{w}{a}=0.70$, where $w$ is the radius at $\frac{1}{e^{2}}$ of the first lobe of the intensity profile, or equivalently $\frac{r_{0}}{a}=0.98$, where $r_{0}$ is the first zero.

\section{EXIN project experimental layout}

The experimental layout is shown in Fig. 4. The electron bunches are generated by the SPARC photo injector with an energy of about $80 \mathrm{MeV}$, a normalized emittance of $1 \mathrm{~mm}$-mrad, a charge of $Q \sim 10$

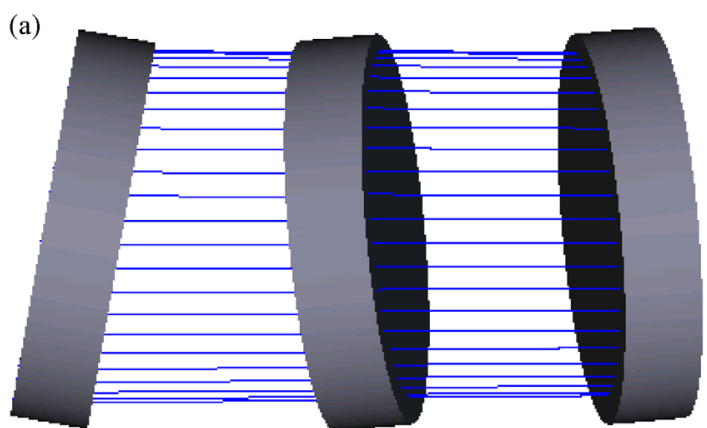

Compensating window system.

(b)

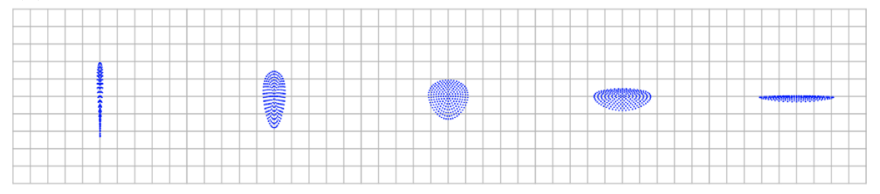

Virtual focus not compensated.

(c)

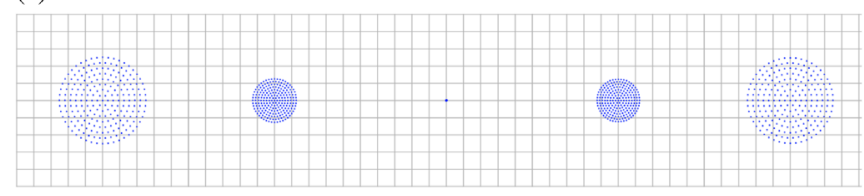

Virtual focus compensated.

Fig. 5. (a) Layout of compensating windows system: part of beam goes through the first mirror after OAP and two windows compensate for aberrations. (c) and (b) Virtual focus with and without compensating windows simulated by ZEMAX.

$\mathrm{pC}$ and a repetition rate of $1-10 \mathrm{~Hz}$. The chosen acceleration regime is the quasi non-linear with normalized laser intensity $a_{0} \sim 1.1$ and a plasma density corresponding to a wavelength $\lambda_{\text {plasma }} \sim 100 \mu \mathrm{m}$. These values determine the range of the laser parameters (energy, temporal length, focal spot) and capillary dimensions to be employed: $(3-4) \mathrm{J}, \quad(30-40) \mathrm{fs}, \quad \sim 100 \mu \mathrm{m}$ and $(120-200) \mu \mathrm{m}$ respectively.

Following the discussion in Section 3 for the best coupling condition of the FLAME laser beam, a focusing element with $f=5 \mathrm{~m}$ (for a capillary diameter of $120 \mu \mathrm{m}$ ) is required. In detail, it would be preferably to employ an off axis parabolic (OAP) mirror in order to avoid any optical aberration and distortion of the laser temporal and spatial profile.

In order to measure the focal spot at each shot, a virtual focus will be provided by focusing a leak (called diagnostic beam "DB") taken from the first mirror after OAP. Since this DB will go through a thick tilted optics while focusing, it will be affected by optical distortions (astigmatism and coma) at the focus point, as simulated with ZEMAX (Fig. 5b). In order to compensate for these distortions and to measure the effective focal spot, a system of compensating windows has been studied in Fig. $5 \mathrm{a}$. By placing two windows as thick as the mirror substrate and tilted with the same angle, it is possible to remove any distortion if they are tilted around the longitudinal axis. From simulations the right tilting angles required to remove the astigmatism and coma are equal to $120^{\circ}$ and $240^{\circ}$ (Fig. 5c) ().

\section{Preliminary alignment test}

Some preliminary tests have been performed in order to study how the laser-capillary coupling can be affected by misalignments, in particular by an offset in the transverse plane (Fig. 7) and 


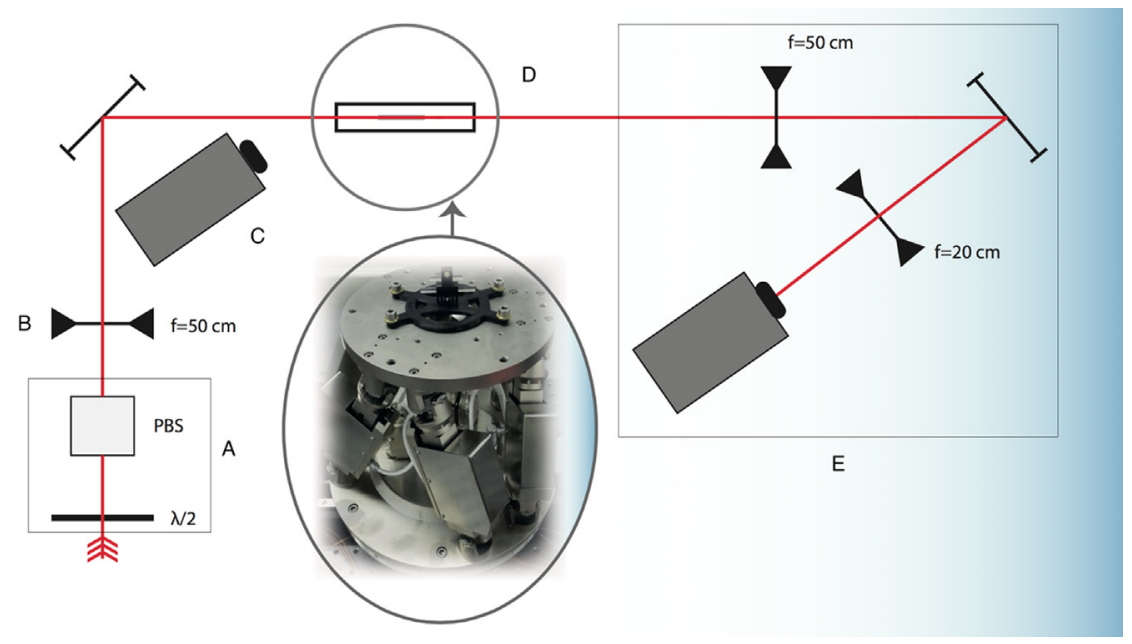

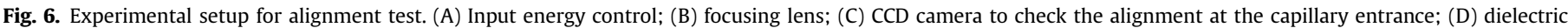
capillary holder sitting on hexapod; (E) imaging system to measure the spot size at the exit of capillary.

(a)

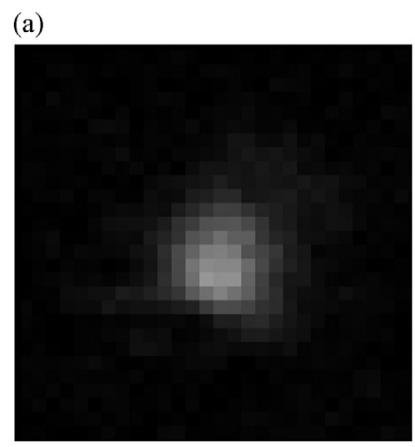

Best coupling with no offset.

(b)

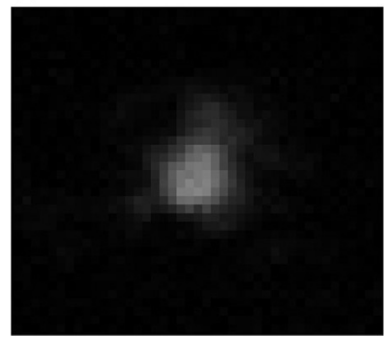

$10 \mu m$ offset.

(d)

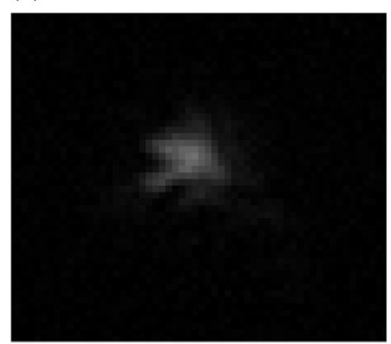

$30 \mu \mathrm{m}$ offset. (c)

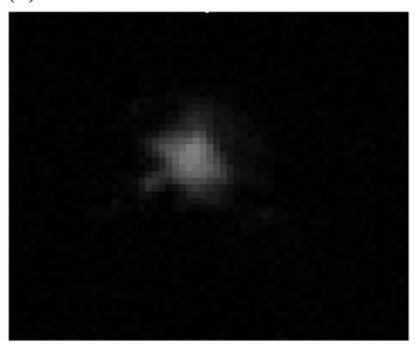

$20 \mu m$ offset.

(e)

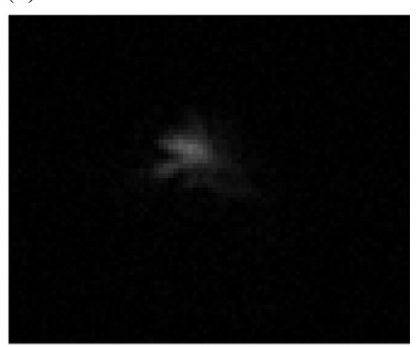

$40 \mu \mathrm{m}$ offset.
Fig. 7. Results of misalignment test with an offset in the transverse plane. (a) Best coupling with no offset, (b) $10 \mu \mathrm{m}$ offset, (c) $20 \mu \mathrm{m}$ offset, (d) $30 \mu \mathrm{m}$ offset, and (e) $40 \mu \mathrm{m}$ offset.

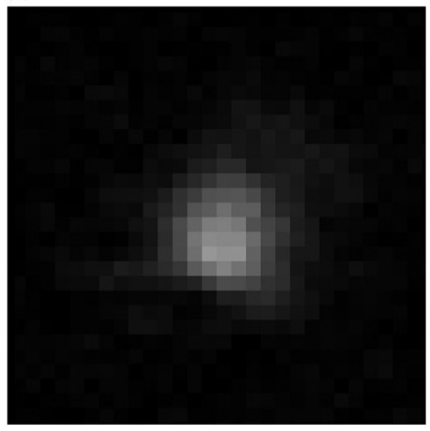

Best coupling with no tilt. (b)

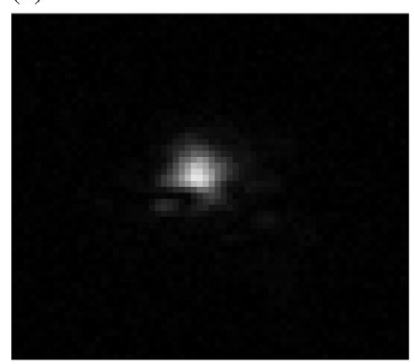

3.5 mrad angular tilt.

(d)

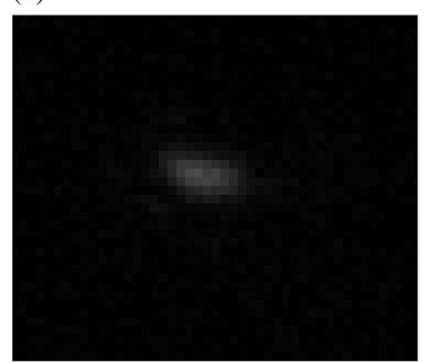

7 mrad angular tilt. (c)

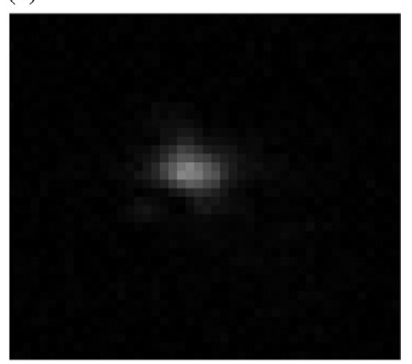

5.2 mrad angular tilt.

(e)

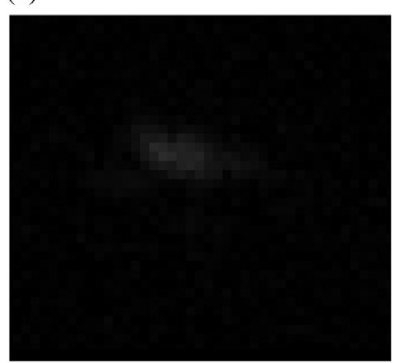

8.7 mrad angular tilt.
Fig. 8. Results of misalignment test with a tilt in the longitudinal plane. (a) Best coupling with no tilt, (b) 3.5 mrad angular tilt, (c) $5.2 \mathrm{mrad}$ angular tilt, (d) $7 \mathrm{mrad}$ angular tilt, and (e) 8.7 mrad angular tilt. 
by a tilt in the longitudinal plane (Fig. 8). Those effects have been measured by moving the PI (Physik Instrumente) hexapod where the capillary was placed. Hexapod platforms are used for precision positioning and alignment of loads in all six degrees of freedom, i.e. three linear axes and three rotational axes.

The experimental setup is shown in Fig. 6. A CW $800 \mathrm{~nm}$, $80 \mathrm{~mW}$ power, p-polarized, $T M_{00}$ mode diode laser has been focused on the capillary entrance and the alignment has been controlled by a CCD camera on the side. The capillary is made in borosilicate with a refractive index for this wavelength equals to $n=1.45$. A two-lenses imaging system has been used to analyse the laser spot at the exit of the capillary. The coupling with the $\mathrm{EH}_{11}$ mode is significantly maintained for a maximal offset of about $20 \mu \mathrm{m}$ (Fig. 7d) and a maximal tilt of about $5 \mathrm{mrad}$ (Fig. 8c).

\section{Conclusions and perspectives}

In this work, the matching conditions required by the EXIN project, to efficiently couple the high power FLAME laser, with its super-Gaussian spatial profile, to a dielectric capillary, have been found. Moreover, a simply and efficient solution in order to have an on-line measurement of the high intensity laser beam have been described and it relies on the possibility to use a virtual focus that can be created by exploiting a mirror leak. A preliminary test has been performed to understand how misalignments can affect laser-capillary coupling. As next step, experimental tests will be performed in order to investigate the coupling of high power laser in the capillary under vacuum condition and then filled with gas. The experimental work will be compared to simulation results. Moreover, since the possibility to characterize the plasma and the wakefield will allow an optimization of the wakefield acceleration process, dedicated studies to develop a proper diagnostic will be carried on. An initial study on effects due to the plasma has been treated in another work [15].

\section{Acknowledgments}

This work has been partially supported by the EU Commission in the Seventh Framework Program, Grant Agreement 312453 EuCARD-2 and by the Italian Ministry of Research in the framework of FIRB-Fondo per gli Investimenti della Ricerca di Base, Project no. RBFR12NK5K.

\section{References}

[1] E. Esarey, P. Sprangle, J. Krall, A. Ting, IEEE Transactions on Plasma Science 24 (1996) 252.

[2] T. Tajima, J.M. Dawson, Physical Review Letters 43 (1979) 267.

[3] W.P. Leemans, et al., Nature Physics 2 (2006) 696.

[4] D.J. Spence, S.M. Hooker, Physical Review E 63 (2001) 015401.

[5] A. Butler, D.J. Spence, S.M. Hooker, Physical Review Letters 89 (2002) 185003.

[6] A.J. Gonsalves, et al., Nature Physics 7 (2011) 11.

[7] E. Esarey, C.B. Schroeder, W.P. Leemans, Reviews of Modern Physics 81 (3) (2009).

[8] W. Leemans, BELLA: multi-GeV electron beam generation and outlook, in: EAAC2015 Conference Communication, 2015.

[9] M. Petrarca, et al., Applied Physics B 114 (2014).

[10] A. Yariv, P. Yeh, Photonics: Optical Electronics in Modern Communications, Oxford University Press, New Delhi, 2007.

[11] B. Cros, C. Courtois, G. Matthieussent, Physical Review E 65 (2002) 026405.

[12] A.R. Rossi, et al., Optimized matching strategy for laser driven plasma boosters, in: Conference Proceedings of EAAC2015, 2015, Submitted to NIMA.

[13] E.G. Neumann, Single-Mode Fibers Springer, Berlin, Heidelberg, (1988).

[14] R.L. Adams, IEEE Journal of Quantum Electronics QE-8 (1972) 838.

[15] A. Curcio, Betatron radiation from capillary tubes, in: Conference Proceedings of EAAC2015, 2015, Submitted to NIMA. 